\title{
ETAPAS INICIAIS NO DESENVOLVIMENTO DE UM MODELO DE PREVISÃO DE ENXOFRE NA DESSULFURAÇÃO DE GUSA EM CARRO TORPEDO NA COMPANHIA SIDERURGICA NACIONAL*
}

\section{Resumo}

\author{
Rafaela Pacheco Malvão dos Santos ${ }^{1}$ \\ Jose Adilson de Castro ${ }^{2}$ \\ André Luiz Vasconcellos da Costa e Silva ${ }^{3}$ \\ Antônio Augusto de Rezende Martins ${ }^{4}$
}

O enxofre no aço é, normalmente, um residual indesejável, afetando negativamente propriedades como ductilidade tenacidade, conformabilidade, soldabilidade e resistência à corrosão. Desde os anos 1970 a prática mais comum é dessulfurar o gusa fora dos altos-fornos, evitando, sempre que possível, a dessulfuração do aço em forno panela. Isto permite vazamento de gusa com teores de enxofre mais elevados do alto-forno, reduzindo os custos de produção desde reator. Termodinamicamente, a dessulfuração do gusa é favorável, devido as condições redutoras e a interação do enxofre com o carbono. Operacionalmente, o gusa pode ser dessulfurado em carros torpedos ou em panelas de transferência, através da adição de diversos reagentes. Para controlar o processo de dessulfuração é necessário conhecer a cinética do processo. Este trabalho descreve estudos iniciais para a melhoria do modelo de controle da dessulfuração em carro torpedo na CSN através da injeção de carbureto de cálcio em pó.

Palavras-chave: Dessulfuração; Enxofre; Gusa; Modelo matemático.

\section{FIRST STEPS IN THE DEVELOPMENT OF A NEW SULPHUR PREDICTION MODEL FOR THE IRON DESULPHURIZATION PROCESS IN THE TORPEDO CAR AT COMPANHIA SIDERÚRGICA NACIONAL}

\section{Abstract}

Sulfur in steel is usually considered an undesirable residual, causing loss of properties such as ductility, toughness, formability, weldability and corrosion resistance. Since the 1970s the practice is to desulphurize the pig iron out of the blast furnaces, producing pig iron with high sulfur but reducing production costs in this reactor. Due to the very low oxygen potential in the pig iron and the carbon sulfur interaction, pig iron desulfurization is thermodynamically very advantageous. This can be done either in torpedo cars or in transfer ladles. In the present work the initial studies to develop an improved model to control de pig iron desulfurization process in the torpedo cars of CSN through the injection of calcium carbide is described.

Keywords: Desulphurization; Sulfur; Pig iron; Mathematical model.

Engenheira Metalurgista, Engenheira de Desenvolvimento Sr., Companhia Siderúrgica Nacional (CSN), Volta Redonda, RJ, Brasil.

2 Engenheiro Metalurgista, Professor Ph.D, Universidade Federal Fluminense (UFF), Rio de Janeiro, RJ, Brasil.

Engenheiro Metalurgista, Professor Ph.D, UFF, Rio de Janeiro, RJ, Brasil.

Engenheiro Metalurgista, Coordenador de Projetos Especiais, Companhia Siderúrgica Nacional (CSN), Volta Redonda, RJ, Brasil. 


\section{INTRODUÇÃO}

Nos últimos anos, observa-se uma crescente demanda de aços com maior tenacidade e ductilidade. Baixos teores de enxofre são importantes para se atingir tais propriedades. Além disto, o enxofre ou os sulfetos podem ter papel importante em alguns processos de corrosão do aço [1]. Assim, na produção de aços para indústrias de tubos e automobilística, por exemplo, o controle do teor de enxofre é primordial. Para tanto é necessário uma elevada eficiência no processo de dessulfuração, com o menor custo possível. A dessulfuração do gusa é, normalmente, uma alternativa barata e eficiente em relação a dessulfuração do aço em forno panela. A dessulfuração do gusa pode ser realizada em panelas de transferência ou no carro torpedo.

Os teores de enxofre final obtidos nesta operação dependem dos agentes dessulfurantes utilizados, das condições termodinâmicas existentes e, principalmente dos aspectos cinéticos do processo. No caso da dessulfuração do gusa a alta temperatura, as principais etapas controladoras do processo estão ligadas aos mecanismos de transferência de massa, já que as reações químicas são bastante rápidas.

Este trabalho visa a contribuir no desenvolvimento de um modelo de predição do enxofre durante o processo de dessulfuração em carro torpedo, permitindo entregar a aciaria um gusa de alta qualidade no menor tempo possível, de forma sistemática e controlada. Para tal, é necessário conhecer o comportamento dos agentes dessulfurantes e a influência dos parâmetros de processo sobre a termodinâmica e a cinética do processo de dessulfuração. Neste trabalho, busca-se estabelecer um modelo para a cinética do processo, que seja eficaz na previsão da evolução do teor de enxofre do gusa em função do tempo e da quantidade de adição de agente dessulfurante.

\subsection{Revisão Bibliográfica}

\subsubsection{Fundamentos cinéticos}

Vários autores modelaram a cinética de injeção de $\mathrm{CaO}$ e $\mathrm{CaC}_{2}$ para a dessulfuração de gusa [2-7]. Neste trabalho preliminar, buscou-se utilizar o modelo mais simples possível para avaliar a sua capacidade preditiva.

De acordo com Deo [8] em um sistema metal-escória-gás onde existem várias fases e interfaces e podem ocorrer reações químicas, os processos podem ser controlados pela transferência de massa em cada uma das fases, pelas reações químicas, por dessorção, por absorção, ou pela combinação destes mecanismos.

Oeters [9] formulou um modelo cinético simples para o processo em que partículas permanecem dispersas e em contato com o metal por um determinado tempo. Segundo Oeters, este processo pode ser dividido em duas partes:

1. A microcinética, que descreve a interação de uma partícula com o metal;

2. A macrocinética que descreve a soma das contribuições das partículas e seu efeito sobre o metal líquido.

Oeters assumiu que o transporte de $S$ no metal controla a absorção de $S$ pela partícula.

Logo, na interface partícula-metal o fluxo de enxofre pode ser descrito por (Equação 1):

$$
j_{S}=\beta_{S}\left[\% S(t)-\% S_{e q}\right](E q .1)
$$


É razoável supor que $\% S_{\text {eq }}$ seja aproximadamente igual a zero no caso da injeção de $\mathrm{CaO}$ ou $\mathrm{CaC}_{2}$, em função dos equilíbrios:

$$
\begin{aligned}
& \mathrm{CaO}+\underline{\mathrm{S}}=\mathrm{CaS}+\underline{\mathrm{O}} \mathrm{e} \\
& \mathrm{CaC}_{2}+\underline{\mathrm{S}}=\mathrm{CaS}+2 \underline{\mathrm{C}}
\end{aligned}
$$

Logo fluxo pode ser aproximado pela (Equação 2):

$$
j_{s}=\beta_{S}[\% S(t)](E q .2)
$$

Onde o coeficiente beta é ajustado para que o fluxo tenha as dimensões gramas $/ \mathrm{m}^{2} . \mathrm{s}$.

Fazendo um balanço de massa do enxofre na partícula, isto é:

[S na partícula em $\mathrm{t}+\mathrm{S}$ que entra na partícula em $\mathrm{dt}=\mathrm{S}$ na partícula em $\mathrm{t}+\mathrm{dt}$ ] É possível obter-se a (Equação 3):

$$
V \rho[S](t)+j A d t=V \rho[S](t+d t)(E q .3)
$$

E, portanto:

$$
j \frac{A}{V \rho}=\frac{d[S](t)}{d t}
$$

Onde [S] é a concentração de enxofre em g de $\mathrm{S} / \mathrm{g}$ de partícula.

Para uma partícula aproximada como uma esfera (Equação 4):

$$
\begin{gathered}
\frac{A}{V}=\frac{4 \pi r^{2}}{\frac{4}{3} \pi r^{3}}=\frac{3}{r}=\frac{6}{d}(E q .4) \\
\beta_{S}[\% S(t)] \frac{6}{d \rho}=\frac{d[S](t)}{d t}(E q .5)
\end{gathered}
$$

Se o enxofre do banho é constante durante o tempo de residência de uma partícula no banho, pode-se integrar a equação (Equação 5) obtendo-se a (Equação 6):

$$
\int_{0}^{t_{R}} \beta_{S}[\% S(t)] \frac{6}{d \rho} d t=\int_{0}^{S_{f}} d[S](t)(E q .6)
$$

A concentração de $S$ final de cada partícula pode ser obtida, portanto, pela Equação 7:

$$
\beta_{S}[\% S(t)] \frac{6}{d \rho} t_{R}=\overline{[S]_{P}}(E q .7)
$$

Esta concentração, multiplicada pelo peso de cada partícula, é o peso de $S$ removido por cada partícula. Ou, multiplicando-se esta concentração pela taxa de injeção $\dot{M}_{P}\left(\frac{g}{s}\right)$, diretamente, pode-se obter a taxa de remoção $\left.\bar{S}\right]_{P} \dot{M}_{P}$.

A análise macrocinética envolve realizar o balanço de massa do enxofre no gusa (Equação 8), que permite obter a (Equação 9): 
[ $S$ no gusa em $t-$ enxofre removido em $d t=S$ no gusa em $t+d t$ ]

$$
\begin{gathered}
\frac{M_{g}}{100} \% S(t)-\overline{[S]_{P}} d t=\frac{M_{g}}{100} \% S(t+d t)(E q .8) \\
-\overline{[S]_{P}} d t=\frac{M_{g}}{100}(\% S(t+d t)-\% S(t)) \\
-\overline{[S]_{P}} d t=\frac{M_{g}}{100}(d \% S(t)) \\
\frac{d \% S(t)}{d t}=-\frac{100}{M_{g}} \overline{[S]_{P}} \quad(E q .9)
\end{gathered}
$$

Substituindo $\overline{[S}_{P}$ (Eq. 9), obtido na análise microcinética, obtem-se a (Equação 10):

$$
\begin{gathered}
-\frac{d \% S(t)}{d t}=\frac{100}{M_{g}} \beta_{S}[\% S(t)] \frac{6}{d \rho} t_{R} \dot{M}_{P} \\
-\frac{d \% S(t)}{\% S(t)}=\frac{100}{M_{g}} \beta_{S} \frac{6}{d \rho} t_{R} \dot{M}_{P} d t \\
\frac{\% S(t)}{\% S(0)}=\exp (-k t) \\
k=\frac{100}{M_{g}} \beta_{S} \frac{6}{d \rho} t_{R} \dot{M}_{P}(E q .10)
\end{gathered}
$$

\section{MATERIAIS E MÉTODOS}

Foram feitos experimentos nas estações de dessulfuração de gusa em carro torpedo da Companhia Siderúrgica Nacional.

Em 35 corridas foram coletadas amostras para análise de enxofre em quatro momentos do processo de dessulfuração, sendo uma amostra inicial, duas intermediárias (com 33\% e 66\% do material injetado) e uma amostra final. O material dessulfurante utilizado em todas as corridas foi carbureto de cálcio $\left(\mathrm{CaC}_{2}\right)$.

A amostragem do gusa, para análise de enxofre, foi realizada manualmente pelo operador da estação de dessulfuração. Esta amostra foi analisada no aparelho Leco. Foram acompanhados durante o experimento, os seguintes parâmetros:

- Peso do gusa a ser dessulfurado;

- Tempo de tratamento;

- Taxa de injeção;

- Teor de enxofre inicial, objetivado e final do gusa;

- Temperatura inicial e final do gusa;

- Consumo de agente dessulfurante;

- Eficiência da dessulfuração;

- Profundidade do sopro de nitrogênio (altura da lança);

— \% silício no gusa.

\section{RESULTADOS E DISCUSSÃO}

Foi utilizado o modelo cinético de Oeters (Equação 20) para ajuste de k (coeficiente cinético global da dessulfuração - Equação 21). 


$$
\begin{gathered}
\frac{\% S(t)}{\% S(0)} \exp (-k t)(E q .20) \\
k=\frac{100}{M_{g}} \beta_{S} \frac{6}{d \rho} t_{R} \dot{M}_{P}(E q .21)
\end{gathered}
$$

A Equação 20 prevê o teor de enxofre em um determinado momento da dessulfuração, a partir do enxofre inicial $(\mathrm{S}(0)$ ), tempo (t) e $\mathrm{k}$ (coeficiente cinético global da dessulfuração).

Observa-se, na Equação 21, que coeficiente cinético global da dessulfuração (k) é diretamente proporcional ao coeficiente de transferência de massa $\left(\beta_{S}\right)$, tempo de residência das partículas no gusa $\left(t_{R}\right)$ e taxa de injeção $\left(\dot{M}_{P}\right)$, e é inversamente proporcional a massa de gusa $\left(M_{g}\right)$ e ao diâmetro da partícula $(d \rho)$.

A primeira tentativa foi ajustar a um único fator $\mathrm{k}$ a todas as experiências. Observouse entretanto, uma dispersão elevada dos resultados conforme Figura 1.

$\mathrm{O}$ fator $\mathrm{k}$ foi ajustado visando minimizar o erro quadrado entre $\mathrm{S} / \mathrm{S} 0$ real (Figura 2) $\mathrm{x}$ $\mathrm{S} / \mathrm{S} 0$ calculado (Figura 3 ). Obteve-se um valor de 0,23. Além da dispersão dos resultados, o erro obtido foi relativamente alto.

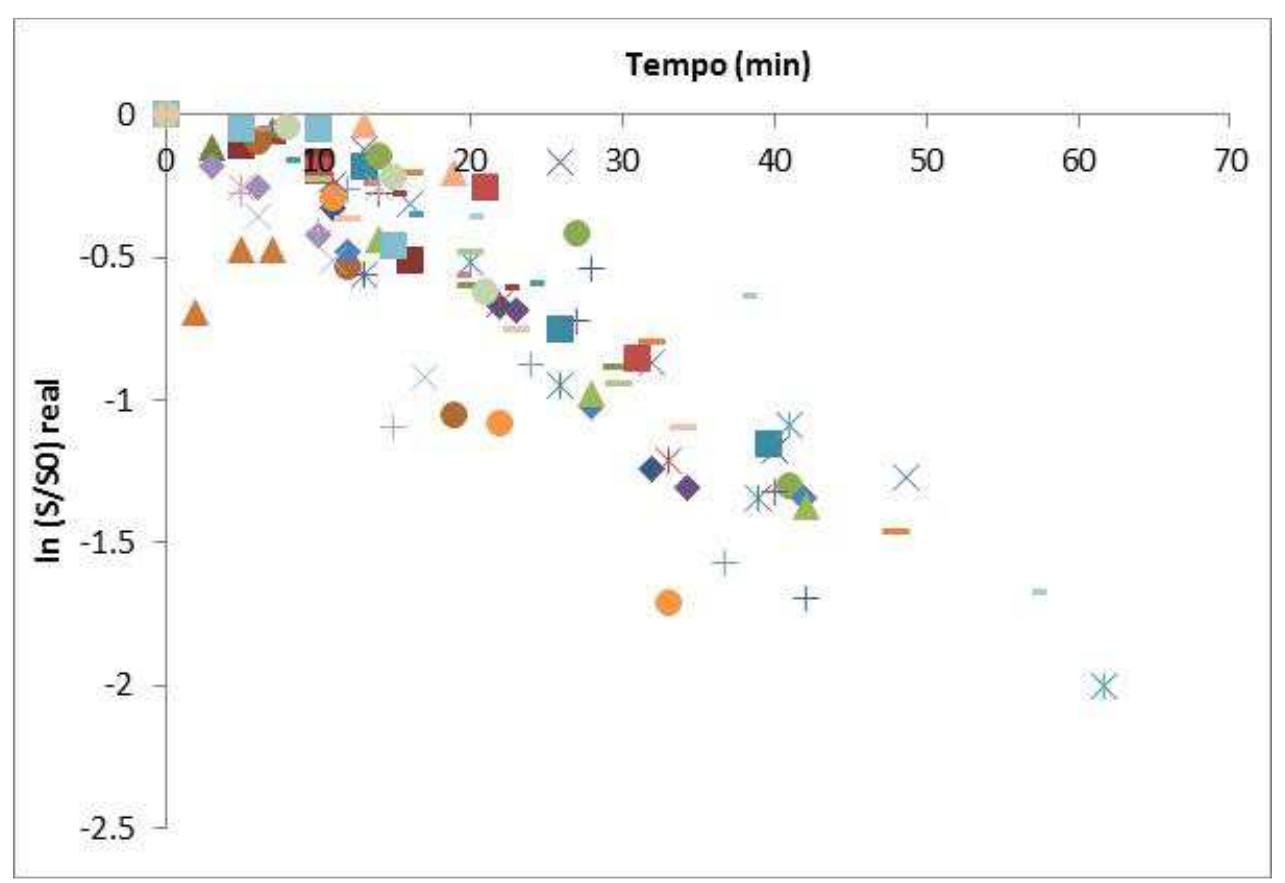

Figura 1 - Resultados de In (S/SO) em função tempo medidos em 4 momentos diferentes da dessulfuração. 


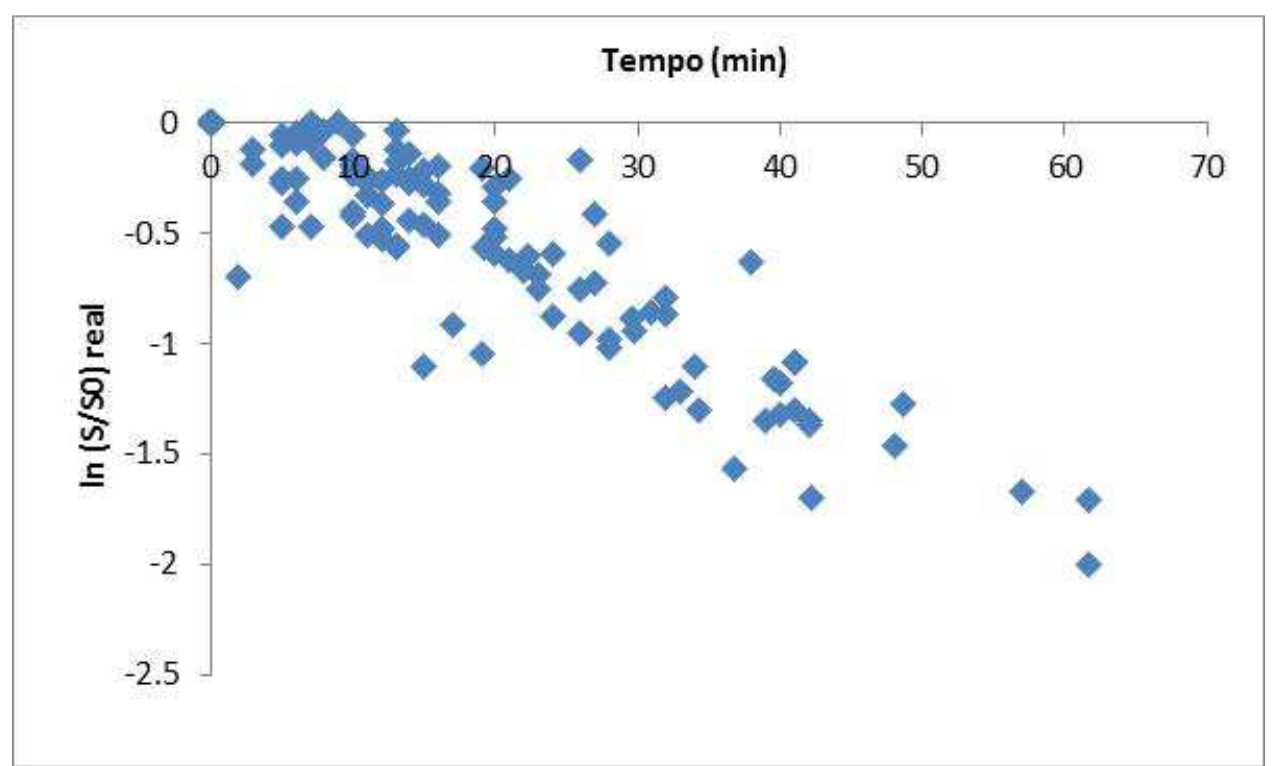

Figura 2 - Resultados de In (S/SO) real em função do tempo medidos em 4 momentos diferentes da dessulfuração.

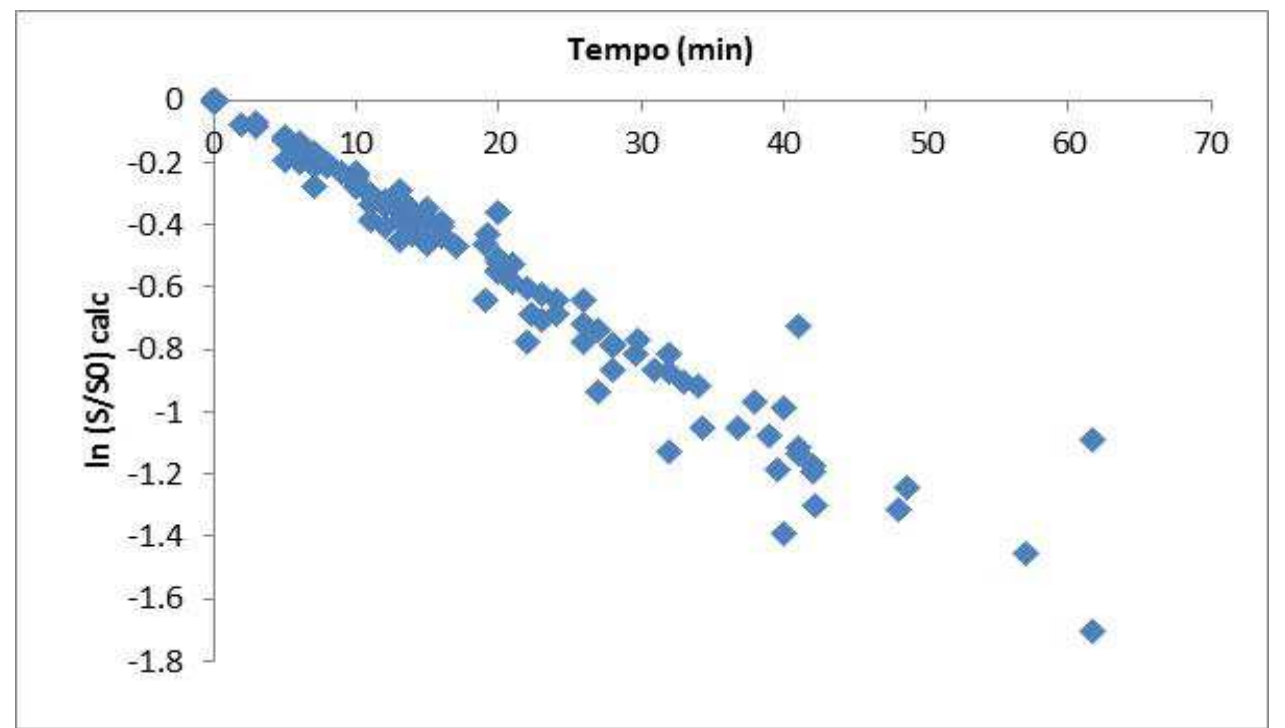

Figura 3 - Resultados de In (S/SO) calculado em função do tempo em 4 momentos diferentes da dessulfuração.

Foram analisadas todas os experimentos de dessulfuração e, pelo seu perfil de queda do teor de enxofre em função do tempo, os dados foram agrupados em 2 grupos, identificados por "círculos" e "quadrados" na figura 4. 


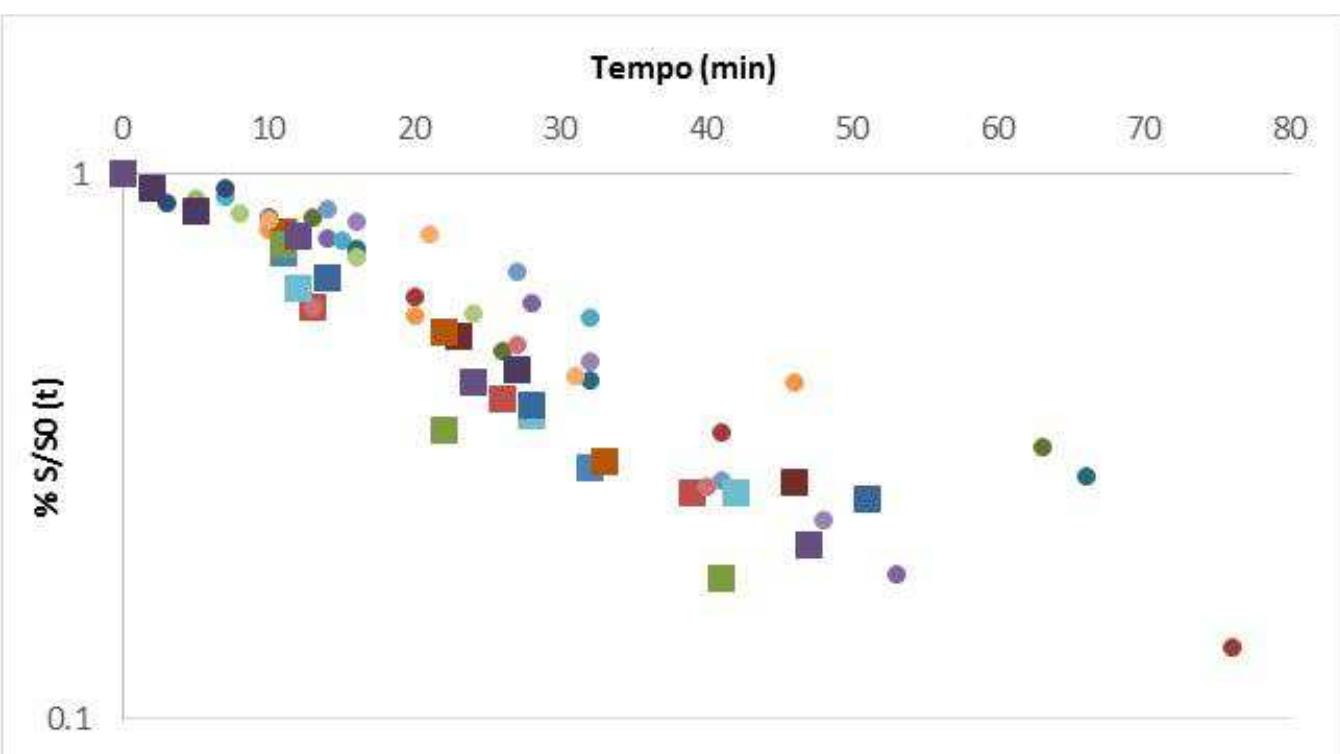

Figura 4 - Agrupamento das dessulfurações em 2 grupos: Cinética mais acelerada e ("quadrados") e mais lenta (("círculos").

Foi levantado o fator $k$ destes grupos "circulos" e "quadrados". Os valores de k obtidos foram de 0,24 e 0,32 respectivamente. Isto mostra claramente que há algumas corridas em que a cinética de dessulfuração é mais rápida do que outras, como mostrado na Figura 5.

Linearizando e separando-se os dados, como mostrado na Figura 6, observa-se que as corridas identificadas pelos "quadrados" tem, de fato, cinética de desulfuração mais favorável. Foram estudadas as variáveis destas dessulfurações e a principal diferença observada foi que estas corridas possuem, em média, um percentual de silício no gusa de $0,64 \%$ e enquanto as corridas onde a desulfuração foi mais lenta, identificadas pelos "circulos", $0,46 \%$. Outra diferença fundamental em relação a estes grupos é que a média de temperatura inicial das corridas de cinética mais rápida é $16^{\circ} \mathrm{C}$ superior a média da temperatura das corridas de cinética mais lenta.

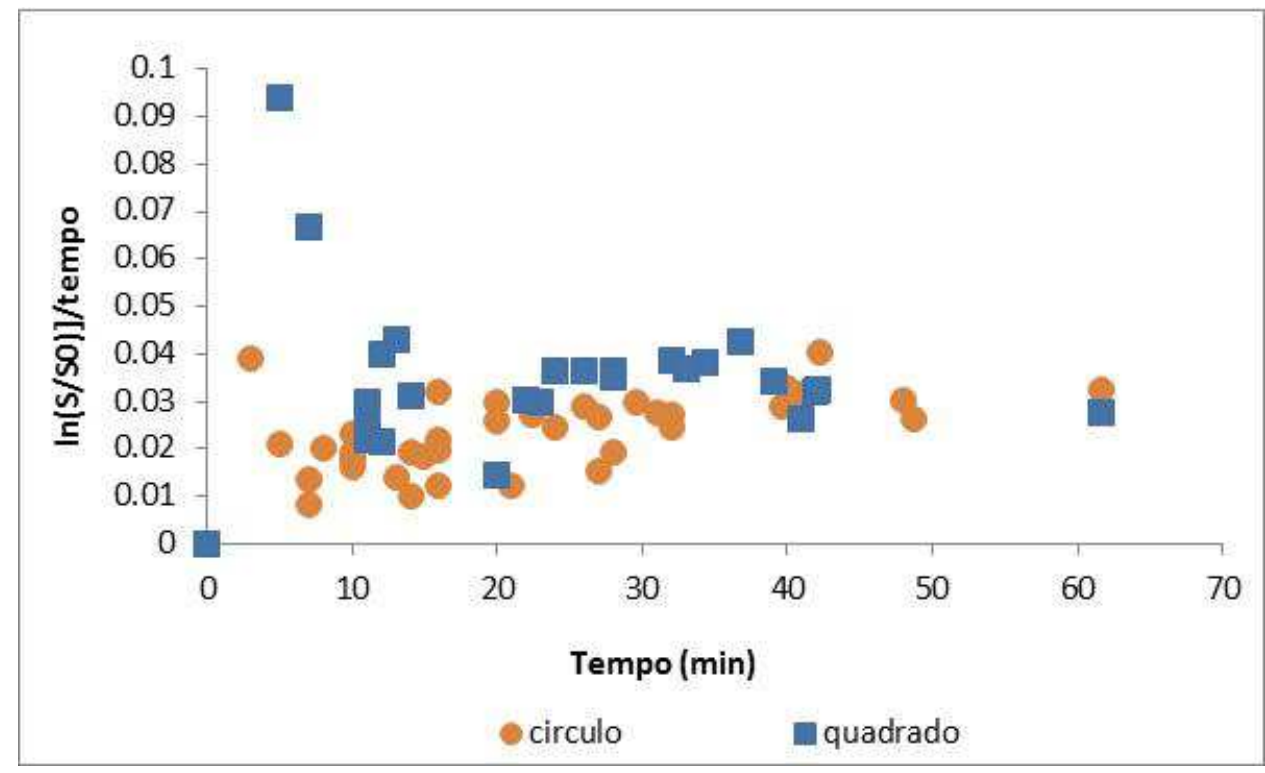

Figura 5 - Valores de $\mathrm{k}$ versus tempo. 


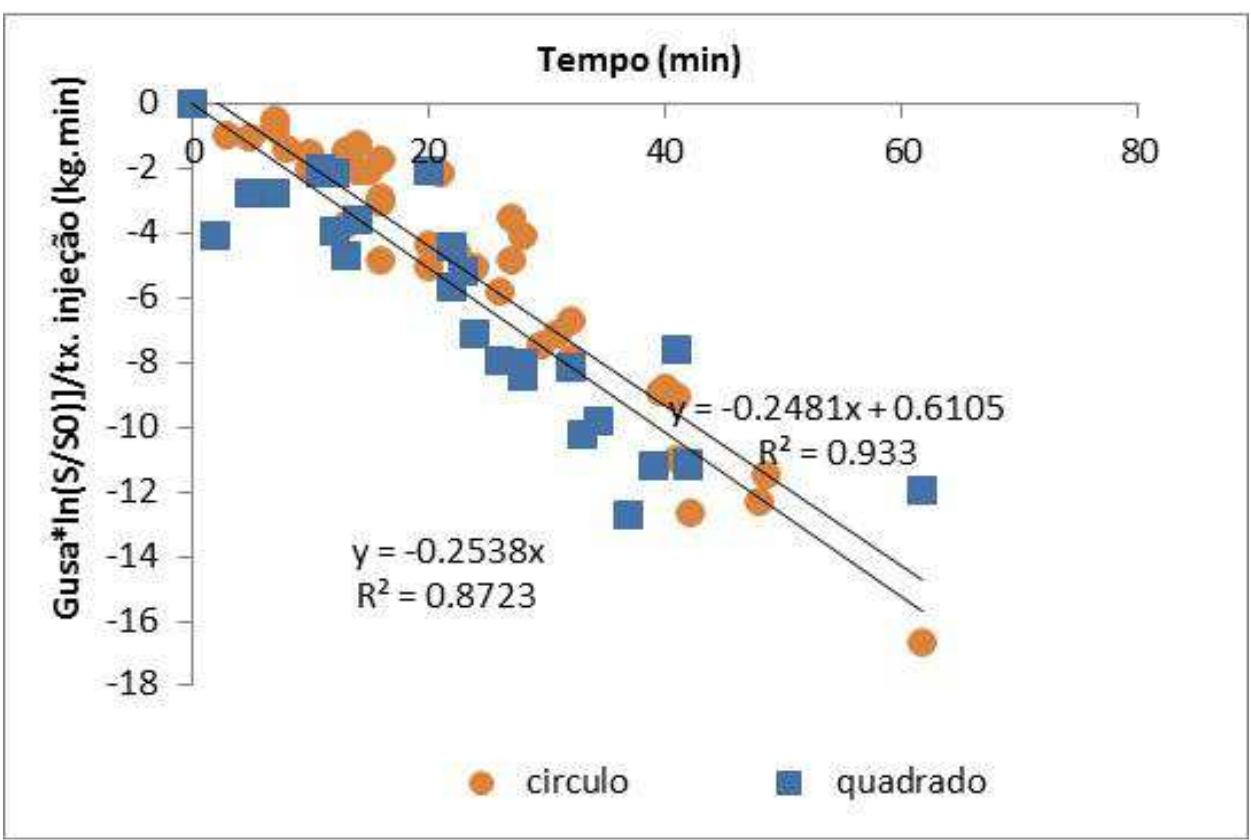

Figura 6 - Valores de kt versus tempo.

\section{CONCLUSÃO}

Para descrever a dessulfuração do gusa com injeção de $\mathrm{CaC}_{2}$ no carro torpedo aplicou-se um modelo simples que considera apenas a reação das partículas com o gusa, adotando várias simplificações. Os resultados indicam que, embora a cinética siga, aproximadamente, o tipo de equação proposto, não parece ser possível descrever satisfatoriamente todas as corridas com a mesma constante cinética. Dois fatores foram identificados como afetando a constante cinética: o teor de Si do gusa e a temperatura de tratamento. As corridas com teor de silício mais alto ou com temperatura mais alta apresentaram cinética mais acelerada de dessulfuração. Isto é razoável em função da interação termodinâmica $S i$ x $S$ e do evidente efeito da temperatura na cinética de transporte. Para contornar este problema o modelo empírico atual empregado na CSN emprega valores de $\mathrm{k}$ diferentes para diferentes faixas de enxofre no gusa. Não foi observado um tempo de incubação para a dessulfuração, como relatado por outros autores. As próximas etapas do modelamento incluem: a análise do equilíbrio termodinâmico metal escória (escória inicial mais carbureto) e a inclusão da possibilidade da dessulfuração ocorrer, simultaneamente, através de reação direta gusa-escória, já proposta por outros autores [2].

\section{REFERÊNCIAS}

1 Pehlke RD, Fuwa T. Control of Sulphur in liquid iron and steel. International Metals Reviews. 1985;30(1):125-40.

2 Varadarajan Seshadri, C A Silva, I A Silva, P. Kroger. A Kinetic model Applied to the Molten Pig Iron Desulfurization by Injection of Lime-based Powders. ISIJ International. 1997;37(1):21-30.

3 Y. Jin, X.G. Bi, S.R. Yu. Kinetic Model For Powder Injection Desulf'urization. Acts Metall Sin (Engl Lett). 2006;19(4):258-64.

4 Zhao Y-F, Irons GA. Calcium carbide powder injection into hot metal Part 2 Simultaneous delsulphurisation and deoxidation. Ironmaking and Steelmaking. 1994;21(4):309-17. 
5 F.Oeters, P.Strohmenger, W.Pluschkell. Desulfurization kinetics of pig iron with lime and natural gas. Archiv fuer das Eisenhuettenwesen. 1973;44(10):727-33.

6 Brodrick C. Desulphurization of pig iron using calcium carbide based reagents. 7thlnt Heavy Minerals Conf "What next", Johannesburg, South Africa [Internet]. 2009 [cited 2015 Apr 1]. p. 141-6. Available from: https://saimm.co.za/Conferences/HMC2009/141146_Broderick.pdf

7 Wei Wu, Zhijun Han, Yanbin Hu, Wei Wu. Desulfurizer desulphurization kinetics by the injection method. Journal of University of Science and Technology Beijing. 2008;15(4):375.

8 Deo, B.; Boom, R. Fundamentals of steelmaking metallurgy. Prentice Hall, 1993.

9 Oeters, F. Kinetic treatment of chemical reactions in emulsion metallurgy. Steel research.Vol.56, n², 1985. 